\title{
THE MODEL OF D-GALACTOSAMINE-INDUCED INJURY OF RAT HEPATOCYTES IN PRIMARY CULTURE
}

\author{
Otto Kučera ${ }^{1}$, Halka Lotková ${ }^{1}$, Roman Kand’ár ${ }^{2}$, Renata Héžová ${ }^{2}$, Vladimíra Mužákováa ${ }^{2}$ Zuzana Červinkováa ${ }^{1}$
}

Charles University in Prague, Faculty of Medicine in Hradec Králové, Czech Republic: Department of Physiology ${ }^{1}$; University of Pardubice, Faculty of Chemical Technology: Department of Biochemical and Biological Sciences ${ }^{2}$

\begin{abstract}
Summary: D-galactosamine (GalN) is a highly selective hepatotoxin that causes liver damage similar to human viral hepatitis via depletion of uridine nucleotides, which subsequently diminishes synthesis of RNA and proteins. Model of galactosamine hepatotoxicity is frequently used in animal experiments in vitro. The purpose of our study was to establish the model of GalN-induced hepatocyte injury in in vitro conditions using primocultures of rat hepatocytes as an important prerequisite for further experiments in which we would like to study potential hepatoprotective effect of various substances. Rate of hepatocyte injury was evaluated by morphological changes, changes in cell viability, albumin production, mitochondrial membrane potential, activity of mitochondrial dehydrogenases and glutathione content. Marked dose dependent hepatocyte injury was found after 24-hour incubation with GalN. Based on the results we suggest as an optimal model for short-term toxicity test exposure to GalN for 24 hours in dose of $40 \mathrm{mM}$.
\end{abstract}

Key words: D-galactosamine; Hepatocytes; Lactate Dehydedrogenase; Lipid Peroxidation; Mitochondrial membrane potential; Albumin synthesis

\section{Introduction}

D-galactosamine (GalN) is frequently used as a model hepatotoxin in animal experiments. Administration of GalN was first described by Keppler et al. (7) to cause a panlobular, focal hepatic necrosis in rats accompanied by microscopic and biochemical features closely resembling those seen in human viral hepatitis. The mechanism by which GalN induces the liver injury is not still completely understood. It is quite clear that galactosamine administration leads to uridine pool depletion resulting in inhibition of messenger RNA synthesis with subsequent depression of protein synthesis $(1,8,9)$. Stachlewitz et al. (18) documented the role of Kupfer cell activation and production of TNF- $\alpha$ as an important event in the mechanism of GalN-induced hepatotoxicity in the rat. They detected apoptosis to occur early after GalN administration before necrosis is developed. Apoptosis was then documented to be caused by activation of caspase-3 (19). Moreover there was reported that hepatotoxic dose of GalN administered to rats induces depletion of hepatic glutathione closely associated with the genesis of the tissue lesion $(11,12)$. The data resulting from experiments using cultured hepatocytes indicate that GalN inhibits synthesis of glutathione due to decreased activity of the enzymes of glutathione synthesis or increased activity of the enzymes involved in glutathione breakdown (14). GalN also decreases NO production in hepatocyte culture by suppressing of inducible nitric oxide synthase (iNOS). This suppression seems to be a consequence of GalN-induced depletion of uridine nucleotides and subsequent general inhibition of total cellular RNA and protein synthesis rather than a specific inhibition of iNOS protein synthesis (15). Thus according to the author's opinion suppression of NO production does not appear to contribute to the toxicity of GalN. GalN toxicity was also not significantly altered by addition of sodium nitroprusside an exogenous donor of NO as demonstrated a study in perfused rat hepatocytes (4). Controversially, Siendones et al. (17) reported that GalN induces cell death by enhancing the production of NO in hepatocyte culture and $\mathrm{PGE}_{1}$ pretreatment reduces GalNinduced cell death through its capacity to reduce iNOS expression and NO production caused by hepatotoxin. Some authors emphasize participation of oxidative stress in pathogenesis of GalN toxicity in vivo $(16,19)$ and in vitro (20).

Nevertheless, testing of various aspects of hepatotoxicity or hepatoprotective mechanisms of different substances using animal experiments exerts certain scientific, ethical and economical objections therefore if possible in vivo experiments should be supplemented by appropriate alternative in vitro method using isolated hepatocytes. Due to the rapid loss of hepatocyte function in cell suspension the studies are performed on freshly isolated cells or short-term monolayer cultures. Three-dimensional techniques (sand- 
wich cultures, bioreactor systems) then allow maintaining hepatocyte functions over an extended period when special environmental conditions mimic those in the intact liver.

The aim of our study was to establish the model of GalN-induced hepatocyte injury in primary culture as a prerequisite for the further studies which will be focused on the evaluation of mechanisms through which hepatotoxic substances including galactosamine influence hepatocyte energy metabolism. This model is also suitable to test potential protective effect of various factors against hepatocyte injury induced by GalN or other hepatotoxins.

\section{Materials and methods}

\section{Chemicals}

Medium William's E without phenolred, foetal bovine serum, penicillin, streptomycin and glutamine were purchased from PAN BIOTECH GmbH (Aidenbach, Germany), type I collagen, trypan blue, Rhodamine 123, kit for lactate dehydrogenase, D-galactosamine, metaphosphoric acid, reduced glutathione, N-ethylmaleimide, o-phthaldialdehyde, sodium phosphate (dibasic, anhydrous), sodium hydroxide, ethylenediaminetetraacetic acid from Sigma Aldrich (St. Louis, MO, USA). Collagenase cruda was obtained from SEVAC (Prague, Czech Republic). Insulin (Actrapid, Hoechst, Frankfurt am Main, Germany), glucagon (Novo Nordisk, Bagsvaerd, Denmark), prednisolon (Solu-Decortin, Merck, NJ, USA), Rat Albumin ELISA Quantification Kit (Bethyl Lab. Inc., Montgomery, Texas), Cell Proliferation Reagent WST - 1 (Roche, Basel, Switzerland) and thiobarbituric acid (Fluka Chemie $\mathrm{GmbH}$, Buchs, Switzerland) were from supplier mentioned in brackets.

Animals
Male albino Wistar rats (BioTest, Konárovice, Czech
Republic) were housed at $23 \pm 1{ }^{\circ} \mathrm{C}, 55 \pm 10 \%$ relative hu-
midity, air exchange $12-14$ times/h, and 12 -hour light-dark
ycle periods (6:00 a.m. to 6:00 p.m.). The animals had free
ccess to standard laboratory rat chow (DOS 2B, Velaz,
Prague, Czech Republic) and tap water. All animals recei-
ed care according to the guidelines set by the institutional
Animal Use and Care Committee of the Charles University,
Prague, Czech Republic.

\section{Hepatocyte Culture and Treatment}

Hepatocytes were isolated from rats mentioned above with the body mass of 230-270 $\mathrm{g}$ by collagenase perfusion (2). The viability of freshly isolated hepatocytes was always more than $90 \%$ as confirmed by trypan blue exclusion. Isolated hepatocytes were suspended in William's E medium supplemented with foetal bovine serum (10\%), glutamine $(2 \mathrm{mM})$, penicillin $(100 \mathrm{IU} / \mathrm{ml})$, streptomycin $(10$ $\mathrm{mg} / \mathrm{ml})$, insulin $(0.08 \mathrm{IU} / \mathrm{ml})$, prednisolon $(0.5 \mu \mathrm{g} / \mathrm{ml})$, glucagon $(0.008 \mu \mathrm{g} / \mathrm{ml})$ and plated in collagen-coated Petri dishes $(60 \mathrm{~mm})$ at a density of $2 \times 10^{6}$ cells / Petri dish.
Hepatocytes were allowed to attach in a gassed atmosphere $\left(5 \% \mathrm{CO}_{2}\right)$ at $37^{\circ} \mathrm{C}$ for 2 hours.

After the establishment of the monolayers, the medium was removed and replaced with fresh medium containing D-galactosamine at different concentrations of 5, 10, 20 and $40 \mathrm{mM}$. Then the hepatocytes were incubated with GalN for $0.5,4$ and 24 hours. Wider range of GalN concentrations were used in 24 hour incubation with this hepatotoxin.

\section{WST-1 assay}

The range of toxic dosage levels of GalN was established by a colorimetric assay based on the cleavage of the tetrazolium salt WST - 1 by mitochondrial dehydrogenases in viable cells. Primary culture of hepatocytes at a density of $3 \times 10^{4}$ cells / well (collagen-coated) was incubated with GalN at the concentrations of 1, 5, 10, 20 and $40 \mathrm{mM}$ for 24 hours. Then all medium was removed, reagent WST - 1 was added (1:20 final dilution) and the cells were incubated in a humidified atmosphere for $120 \mathrm{minu}-$ tes. The absorbance of the samples was measured using a microtiter plate reader Multiskan at dual wavelength mode of 450 and $690 \mathrm{~nm}$ respectively.

\section{Mitochondrial membrane potential}

Changes in mitochondrial membrane potential were evaluated from uptake of Rhodamine 123 - a cationic fluorescent probe, which accumulates into energised mitochondria (3). Hepatocyte cultures (gassed with $5 \% \mathrm{CO}_{2}$ ) were incubated with $60 \mathrm{nM}$ Rhodamine 123 at $37^{\circ} \mathrm{C}$ for $15 \mathrm{~min}$. Then the medium was removed and centrifuged (50 x g). Rhodamine concentration was determined in this medium using Perkin-Elmer Luminiscence Spectrometer LS 50B (Perkin Elmer Inc, Boston, MA, USA) at excitation and emission wavelengths of 498 and $525 \mathrm{~nm}$.

\section{Biochemical Assays}

Hepatocyte toxicity was analysed by lactate dehydrogenase activity (LDH) in the culture medium using a commercial kit from Sigma - Aldrich.

Concentration of malondialdehyde (MDA) was measured as TBARS in the culture medium and served as a marker of lipid peroxidation.

Antioxidative conditions of hepatocytes were determined by the measurement of glutathione content. Reduced form of glutathione (GSH) was analysed by reverse-phase high-performance liquid chromatography. Samples were prepared using slightly modified method of Hissin et Hilf (5). HPLC analysis was performed using an autosampler SIL-10ADvp, a pump LC-10ADvp, a column oven CTO-10 ACvp, a fluorescence detector RF-10Axl and a system controller SCL-10Avp (Shimadzu, Kyoto, Japan). The reversephase column Discovery C18, $15 \mathrm{~cm}$ x $4 \mathrm{~mm}, 5 \mu \mathrm{m}$ (Supelco, Bellefonte, PA, USA) served as separation. The mixture of methanol and $0.25 \mathrm{mM}$ sodium phosphate adjusted to $\mathrm{pH}$ 6.0 was used as mobile phase. Twenty $\mu 1$ of sample was in- 
jected on the column $\left(37^{\circ} \mathrm{C}\right)$. Fluorometric detection (excitation wavelength $350 \mathrm{~nm}$, emission wavelength $420 \mathrm{~nm}$ ) followed a postcolumn derivatization reaction with o-phthalaldehyde. The CSW32 system (DataApex, Prague, Czech Republic) was used for collecting and processing of the chromatographic data. The amount of GSH was quantified from the corresponding peak area.

\section{Albumin measurement by ELISA}

To evaluate functional capacity of cultured hepatocytes the amount of albumin secreted into the culture medium during incubation period was measured using the commercial kit from Bethyl Lab. Inc., USA.

\section{Light Microscopy}

The morphological changes of hepatocytes in culture were examined using inverted microscope with phase contrast Olympus CK 40 (Tokyo, Japan). Microphotographs were obtained by digital camera Olympus Camedia C 4040.

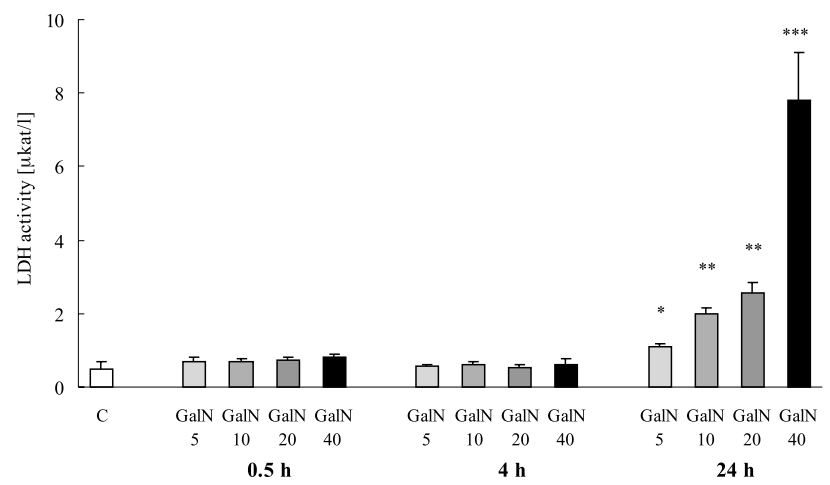

Fig. 1: Effect of GalN treatment in concentrations of 5, 10, 20 and $40 \mathrm{mmol} / 1$ for $0.5,4$ and 24 hours on the release of LDH from hepatocytes. Each column represents the mean value and bars the standard deviation $(n=3) .{ }^{*} \mathrm{p}<0.05$, $* * p<0.01, * * * p<0.001$ compared with controls.

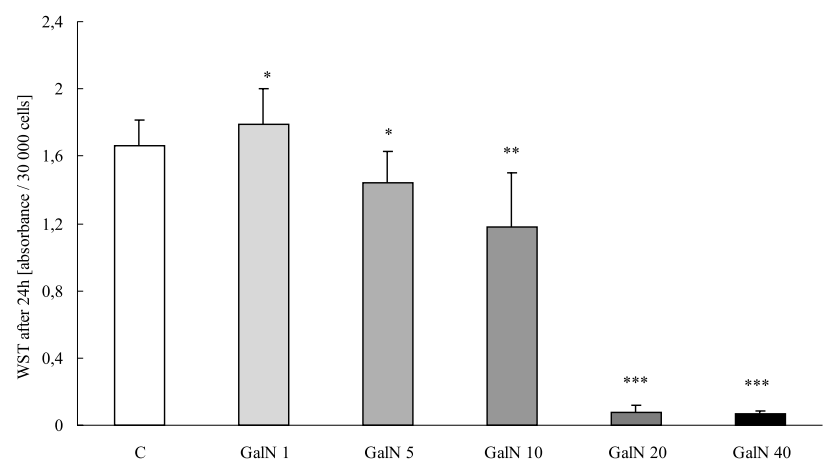

Fig. 3: WST-1 assay as a test of cell viability after treatment of rat hepatocyte primoculture with GalN in concentrations of 1, 5, 10, 20 and $40 \mathrm{mmol} / 1$ for 24 hours. Each column represents the mean value and bars the standard deviation $(n=8) .{ }^{*} p<0.05, * * p<0.01, * * * p<0.001$ compared with controls.

\section{Statistical analysis}

Due to normal variation among different primary culture preparations the results of a typical experiment are expressed. Experiments were repeated tree times using different hepatocyte preparations. Similar trends were observed in each experiment. All values are expressed as means \pm SD. The statistical significance was analysed using ANOVA test and judged as $\mathrm{p}^{*}<0.05, \mathrm{p}^{* *}<0.01, \mathrm{p}^{* * *}<0.001$ respectively.

\section{Results}

\section{Cytotoxic effect of D-Galactosamine}

LDH activity in culture media is one of the most important markers of hepatocyte injury. We did not find any significant change in LDH activity in hepatocyte cultures exposed to GalN for 0.5 and 4 hours respectively. As documented in Fig. 1 and 2 significant dose-dependent increase in LDH activity appears after $24 \mathrm{~h}$ incubation with GalN.

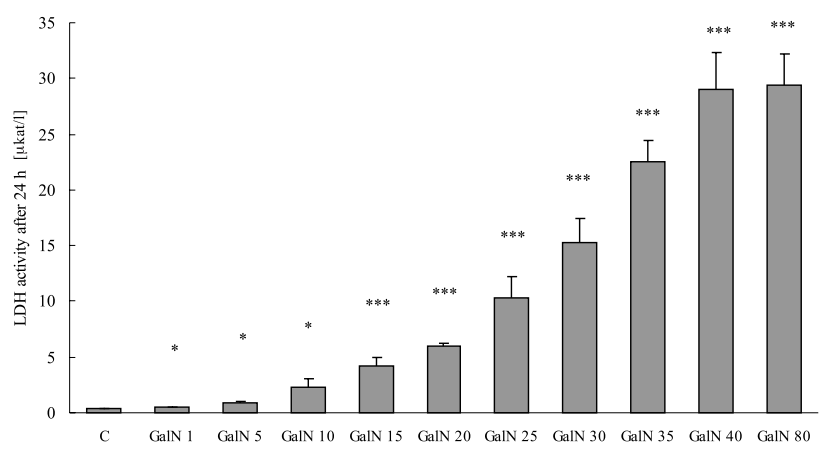

Fig. 2: Effect of GalN treatment in concentrations of 1, 5, $10,15,20,25,30,35,40$ and $80 \mathrm{mmol} / 1$ for 24 hours on the release of $\mathrm{LDH}$ from hepatocytes. Each column represents the mean value and bars the standard deviation $(n=3)$. ${ }^{*} \mathrm{p}<0.05, * * * \mathrm{p}<0.001$ compared with controls.

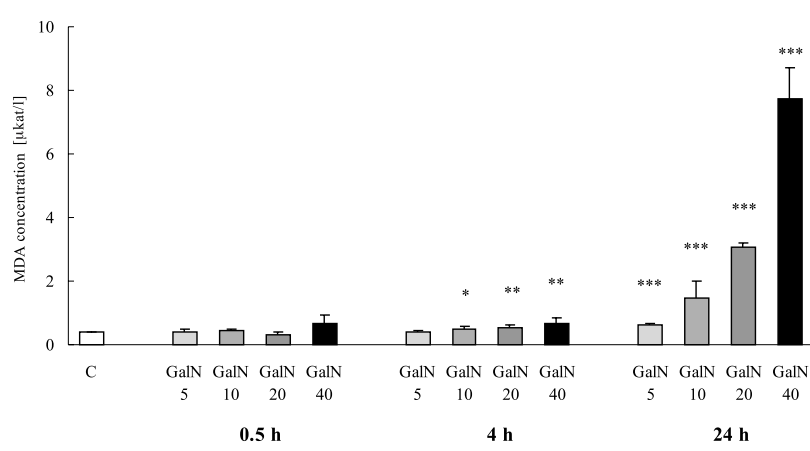

Fig. 4: Effect of GalN treatment in concentrations of 5, 10, 20 and $40 \mathrm{mmol} / 1$ for $0.5,4$ and 24 hours on production of MDA. Each column represents the mean value and bars the standard deviation $(n=3)$. ${ }^{*} \mathrm{p}<0.05,{ }^{* *} \mathrm{p}<0.01, * * * \mathrm{p}<0.001$ compared with controls. 
With respect to the results of LDH activity all other parameters were measured after 24 hours incubation with GalN.

\section{WST-1 Assay}

The toxic effect of GalN in the doses of 1, 5, 10, 20, and $40 \mathrm{mM}$ was tested by WST- 1 assay. In this test an expansion in the number of viable cells results in an increase of the activity of mitochondrial dehydrogenases and consequently in the amount of formazan dye quantified by measuring the absorbance of the dye. While the lowest concentration of GalN ( $1 \mathrm{mM}$ ) did not exhibit any toxic effect on hepatocytes, higher concentrations led to decrease of the activity of mitochondrial dehydrogenases in a dose dependent manner thus documenting the toxic effect of GalN (Fig. 3).

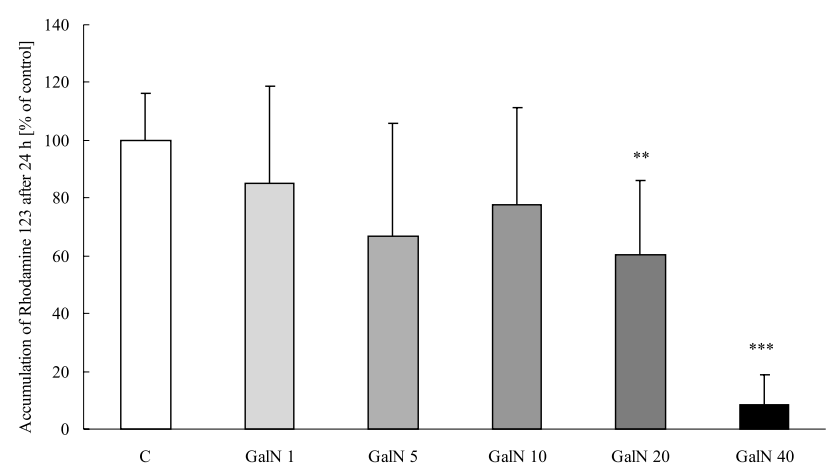

Fig. 5: Effect of GalN treatment in concentrations of 1, 5, 10,20 and $40 \mathrm{mmol} / 1$ for 24 hours on accumulation of Rhodamine 123 by hepatocytes. Accumulation of Rhodamine 123 by control hepatocyte groups is expressed as $100 \%$. Each column represents the mean value and bars the standard deviation $(\mathrm{n}=8) .{ }^{* *} \mathrm{p}<0.01,{ }^{* *} \mathrm{p}<0.001$ compared with controls.

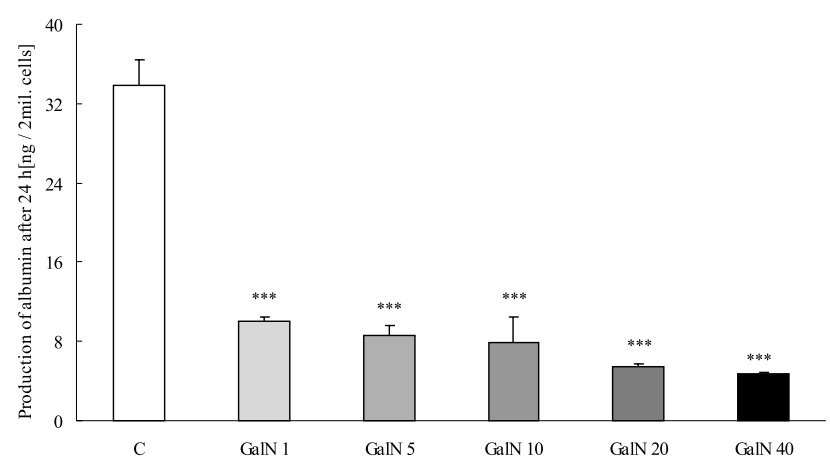

Fig. 7: Effect of GalN in concentration of 1, 5, 10, 20 and 40 $\mathrm{mmol} / 1$ on production of albumin in rat hepatocyte primoculture after 24 hours. Each column represents the mean value and bars the standard deviation $(\mathrm{n}=3) . * * \mathrm{p}<0.001$ compared with controls.

\section{Effect of GalN on lipid peroxidation}

Fig. 4 documents that galactosamine produced dose and time dependent increase in concentration of MDA, which serves as a marker of lipid peroxidation. The incubation of hepatocytes with GalN for $0.5 \mathrm{~h}$ did not cause any significant change in lipid peroxidation. Except for the lowest dose, GalN induced the production of MDA at all of used concentrations after the incubation period of $4 \mathrm{~h}$. The maximal production of MDA was seen when hepatocytes were incubated with GalN for $24 \mathrm{~h}$.

\section{Effect of GalN on mitochondrial membrane potential}

In intact mitochondria Rhodamine 123 distributes electrophoretically into the mitochondrial matrix. Low accumulation of Rhodamine 123 in the mitochondria shows for the decrease in mitochondrial membrane potential that was

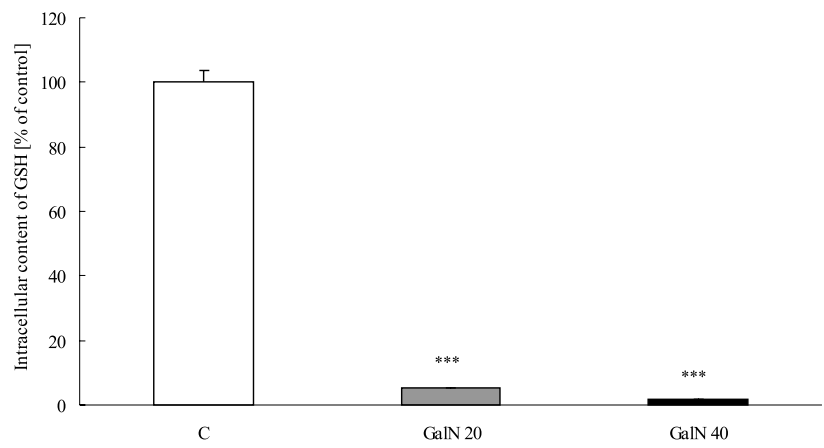

Fig. 6: Effect of GalN in concentration of 20 and $40 \mathrm{mmol} / 1$ on intracellular GSH content in rat hepatocyte primoculture after 24 hours. Each column represents the mean value and bars the standard deviation $(\mathrm{n}=3) .{ }^{* * *} \mathrm{p}<0.001 \mathrm{com}-$ pared with controls

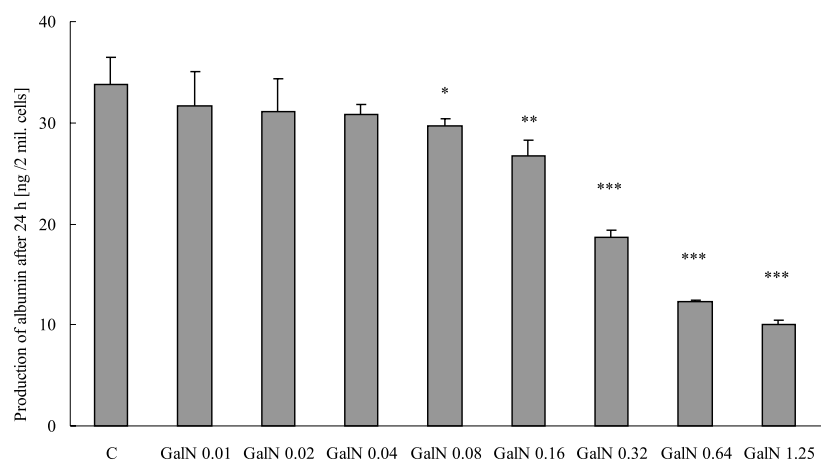

Fig. 8: Effect of GalN in concentration of 0.01, 0.02, 0.04, $0.08,0.16,0.32,0.64$ and $1.25 \mathrm{mmol} / 1$ on production of albumin in rat hepatocyte primoculture after 24 hours. Each column represents the mean value and bars the standard deviation $(\mathrm{n}=3) .{ }^{*} \mathrm{p}<0.05,{ }^{* *} \mathrm{p}<0.01, * * * \mathrm{p}<0.001 \mathrm{com}-$ pared with controls. 
documented after incubation with 20 and $40 \mathrm{mM}$ GalN for 24 hours (Fig. 5). GalN at concentrations of 1, 5 and $10 \mathrm{mM}$ did not produce a significant decline in mitochondrial membrane potential.

\section{Effect of GalN on the depletion of glutathione}

Incubation with both 20 and $40 \mathrm{mM}$ GalN for $24 \mathrm{~h}$ caused a statistically significant decrease in GSH as documented in Fig. 6.
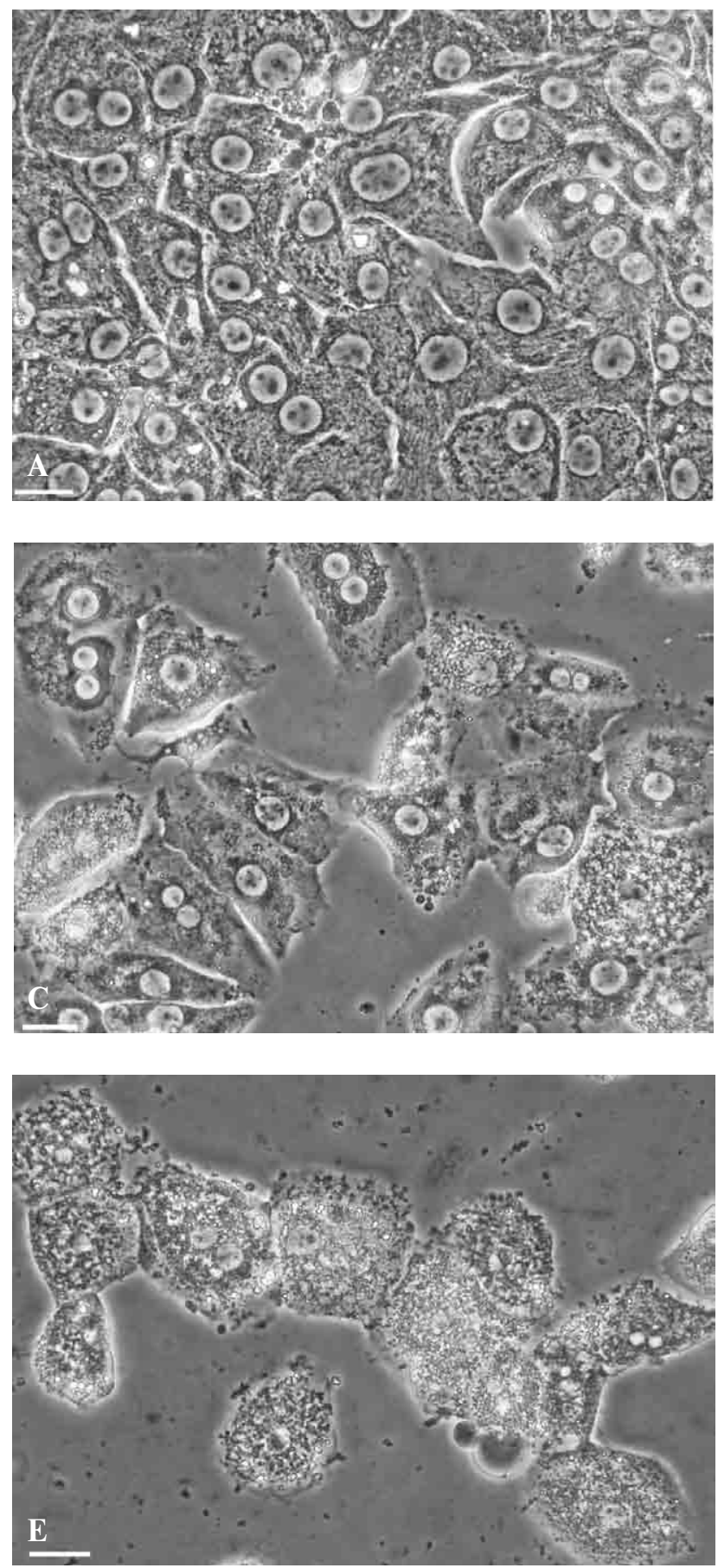

\section{Effect of GalN on albumin production}

Fig. 7 documents the production of albumin by hepatocytes incubated with GalN for $24 \mathrm{~h}$ at concentrations used previously for testing of other parameters $(1,5,10,20$ and $40 \mathrm{mM}$ ). The production was dramatically reduced for all of used concentrations. Then the test of albumin production was carried out for lower concentrations as documented in Fig. 8. The albumin production decreased dose dependently, the significant decrease was discovered when
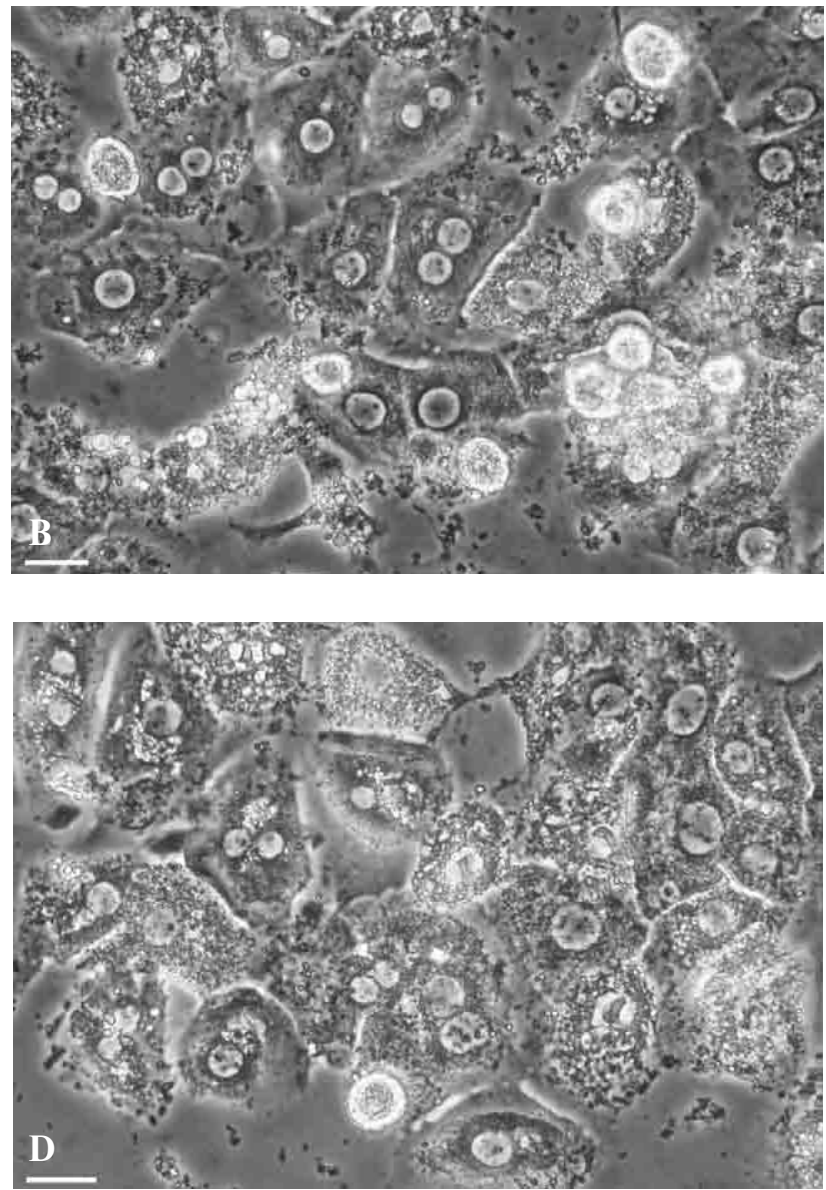

Fig. 9A,B,C,D,E: Microphotographs of control rat hepatocyte primoculture (A) and primary culture incubated with GalN in concentrations of 5 (B), 10 (C), 20 (D) and 40 (E) $\mathrm{mmol} / 1$ for 24 hours (phase contrast, magnification 400x for all microphotographs, bar $10 \mu \mathrm{m}$ ). 
hepatocytes were incubated with GalN at the concentrations of $0.08 \mathrm{mM}$ and higher.

\section{Morphological changes in culture}

Incubation of hepatocytes with GalN was associated with discontinuities of the plasma membrane, highly granular cytoplasm, marked reduction in brightness contrast between the nucleus and cytoplasm. The shape of hepatocytes was more spherical in comparison with controls (Fig. 9A). These changes were intensified using higher concentrations of GalN (Fig. 9B,C,D,E). While the viability of control hepatocytes was $97 \%$ as measured by trypan blue exclusion test at the end of the cultivation period, increase in the concentration of GalN led to fall in viability so that the hepatocytes incubated with $40 \mathrm{mM}$ GalN did not exert any viability.

\section{Discussion}

Different hepatotoxic agents - acetaminophen, ethanol, carbon tetrachloride, thioacetamide or galactosamine are frequently used as model substances that induce liver injury in animals. Due to scientific, ethical and also economical reasons there is a high tendency to replace animal models by appropriate alternative methods. Isolated hepatocytes fulfil these requirements. Therefore we established the standard procedure of preparation of isolated rat hepatocytes and the model of primary hepatocyte culture. Cell culture model then allows evaluating the mechanisms through which hepatotoxic substances mentioned above can influence hepatocyte energy metabolism since the availability of energy is one of the important factors modifying liver injury. Knowledge of these mechanisms could help us to find effective protection against hepatocyte injury. The mechanisms of liver injury induced by hepatotoxins may differ. GalN is reported to cause liver damage similar to human viral hepatitis via depletion of uridine nucleotides and, subsequently diminishing synthesis of RNA and proteins as mentioned in introduction. Unfortunately, only a few reports documenting GalN-induced injury to isolated hepatocytes are available. Moreover there is growing evidence that mitochondria play an important role in pathogenesis of hepatocyte injury induced by different hepatotoxins (13). Mitochondrial permeability transition (MPT) leading consequently to dissipation of mitochondrial membrane potential is considered as a mechanism of both, apoptosis or necrosis, depending on the extent of ATP loss (10). Apoptotic death may be mediated by the release of apoptosis-inducing factor or of cytochrome c leading to caspase activation. With respect to the finding of Stachlewitz et al. (18) that GalN-induced necrosis is preceded by TNF $\alpha$-mediated apoptosis the effect of GalN on mitochondria seems to be evident. Therefore the aim of this study was to establish the model of GalN-induced hepatocyte injury in primary culture with regard to its effect on mitochondria as an essential prerequisite for our further studies.

The cytotoxicity induced by GalN was evaluated by measuring of LDH activity in cultivation medium. While the incubation of hepatocytes with GalN did not cause an increase in LDH activity after 0.5 or $4 \mathrm{~h}$ incubation, GalN at all used concentrations $(5,10,20$ and $40 \mathrm{mM})$ induced a dose dependent significant increase in LDH activity after 24 hours incubation (Fig. 1, 2). Uridine pool depletion and inhibition of protein synthesis were reported to cause necrosis of the liver after GalN administration as mentioned above. Sun et al. (19) documented that GalN-induced lipid peroxidation and cell necrosis in the liver were the results of oxidative stress. In our experiment GalN-induced lipid peroxidation was observed 4 hours after the incubation with GalN at concentrations of 10, 20 and $40 \mathrm{mM}$ (Fig. 4). Lipid peroxidation was further pronounced in dose dependent manner when hepatocytes were incubated with GalN for 24 hours. The cytotoxic effect of GalN paralleled its effect on lipid peroxidation only after $24 \mathrm{~h}$ incubation so that the lipid peroxidation seems to be important but not the main cause of GalN-induced hepatocyte death. The genesis of the tissue lesion after GalN administration to rats is associated with the loss of hepatic glutathione as reported by MacDonald et al. $(11,12)$. With respect to the fact that the cytotoxicity of GalN is mostly pronounced when the concentration of 20 and $40 \mathrm{mM}$ was added to the culture media for 24 hours, GSH content was measured using these concentrations and incubation period. GSH content in cells was dramatically reduced (Fig. 6). McMillan and Jollow (14) detected the reduction of GSH content even if the hepatocyte cultures were exposed to small concentration ( $4 \mathrm{mM}$ ) of GalN.

The effect of GalN on mitochondria was evaluated by the method according to Emaus et al. (3) based on the accumulation of Rhodamine 123 into viable mitochondria. Low accumulation of Rhodamine 123 gives evidence for the decrease in the mitochondrial membrane potential that was measured after $24 \mathrm{~h}$ incubation of hepatocytes with 20 and $40 \mathrm{mM}$ GalN (Fig. 5). Lower concentrations of GalN did not cause a significant change of rhodamine accumulation. Thus we can assume that GalN-induced hepatotoxicity is at least partly associated with a loss of mitochondrial function. GalN is known to deplete uridine pool in hepatocytes, which leads to the inhibition of proteosynthesis (8). Albumin synthesis is widely used marker for testing of hepatocyte functional capacity. Impaired albumin production in GalN-treated primary rat hepatocyte cultures was confirmed by Ishizaki-Koizumi et al. (6). Our results determined the dose dependent fall in albumin that resulted when using very low concentrations of GalN $(0.08 \mathrm{mM})$ and higher (Fig. 7, 8). Interestingly, the production of albumin could be more sensitive marker of hepatocyte function than urea synthesis. Incubation of hepatocytes even with the dose of $40 \mathrm{mM}$ GalN did not cause decrease in 
urea production during the first 24 hours of incubation (data not shown).

We successfully established in vitro model of GalN-induced injury suitable for our further experiments. These experiments are primarily focused on studies determining the mechanisms of protection against hepatocyte injury induced by different hepatotoxins including GalN. Evaluation of energetic metabolism using this model should elucidate the effect of GalN on mitochondria and availability of energy respectively - the critical steps of cell injury and prevention from it.

\section{Conclusions}

Our study demonstrates the short-term model of GalNinduced hepatocyte injury in primary culture. The incubation of hepatocytes with GalN at the dose of $40 \mathrm{mM}$ for 24 hours allows GalN to exert its cytotoxic effect associated with lipid peroxidation, decrease in GSH content and mitochondrial injury.

\section{Acknowledgement}

This work was supported by research project MSM 0021620820 .

\section{References}

1. Anukarahanonta $\mathrm{T}$, Shinozuka $\mathrm{H}$, Fearber E. Inhibition of protein synthesis in rat liver by D-galactosamine. Res Commun Chem Pathol Pharmacol 1973:5:481-91.

2. Berry MN, Edwards AM, Barritt GJ. Isolated hepatocytes preperation, properties and application. In: Burdon RH, van Knippenberg PH, eds. Elsevier, New York, Oxford, Amsterdam, 1991:15-81.

3. Emaus RK, Grunwald R, Lemasters JJ. Rhodamine 123 as a probe of transmembrane potential in isolated rat-liver mitochondria: spectral and metabolic properties. Biochim Biophys Acta 1986;850:436-48

4. Farghali H, Zidek Z, Hynie S. Effects of nitroprusside as a nitric oxide donor on anoxia/reoxygenation and D-galactosamine hepatic injuries: a study in perfused hepatocytes. Physiol Res 1997;46:363-9.

5. Hissin PJ, Hilf R. A fluorometric method for determination of oxidized and reduced glutathione in tissues. Anal Biochem. 1976;74:214-26.

6. Ishizaki-Koizumi S, Sonaka I, Fujitani S, Nishiguchi S. Mechanisms of the protective effect of L-alanine to D-galactosamine-induced hepatocellular injury: comparative studies of L-alanine and pyruvate. Biochem Biophys Res Commun 2002;291:738-43.

7. Keppler D, Lesch R, Reutter W, Decker K. Experimental hepatitis induced by D-galactosamine. Exp Mol Pathol 1968;9:279-90.
8. Keppler D, Rudigier J, Bischoff E, Decker K. The trapping of uridine phosphates by D-galactosamine, D-glucosamine, and 2-deoxy-D-galactose. Eur J Biochem 1970;17:246-53.

9. Keppler D, Pausch J, Decker K. Selective uridine triposphate deficiency induced by D-galactosamine in liver and reversed by pyrimidine nucleotide precursors: Effect on ribonucleic acid synthesis. J Biol Chem 1974;249:211-16.

10. Lemasters JJ, Nieminen AL, Qian T, Trost LC, Elmore SP, Nishimura Y, Crowe RA, Cascio WE, Bradham CA, Brenner DA, Herman B. The mitochondrial permeability transition in cell death: a common mechanism in necrosis, apoptosis and autophagy. Biochim Biophys Acta. 1998;1366:177-96.

11. MacDonald JR, Gandolfi AJ, Sipes IG. Cystamine modulation of galactosamineinduced hepatotoxicity. Toxicol Appl Pharmacol 1984;73:551-58.

12. MacDonald JR, Gandolfi AJ, Sipes IG. Hepatic cysteamine and nonprotein sulfhydryl levels following cystamine or cysteamine treatment of galactosamine-poisoned rats. Drug Chem Toxicol 1985;8:483-94.

13. Masubuchi Y, Nakayama S, Horie T. Role of mitochondrial permeability transition in diclofenac-induced hepatocyte injury in rats. Hepatology 2002;35:544-51.

14. McMillan JM, Jollow DJ. Galactosamine Hepatotoxixity: Effect of galactosamine on glutathione resynthesis in rat primary hepatocyte cultures. Toxicol Appl Pharmacol 1992;115:234-40.

15. McMillan JM. Galactosamine decreases nitric oxide formation in cultured rat hepatocytes: Lack of involvement in cytotoxicity. J Biochem Toxicol 1999;13: 143-8.

16. Quintero A, Pedraza CA, Siendones E et al. PGE1 protection against apoptosis induced by D-galactosamine is not related to the modulation of intracellular free radical production in primary culture of rat hepatocytes. Free Radic Res 2002, $36: 345-55$

17. Siendones E, Fouad D, Abou-Elella A, Quintero A, Barrera P, Muntané J. Role of nitric oxide in D-galactosamine.induced cell death and its protection by $\mathrm{PGE}_{1}$ in cultured hepatocytes. Nitric Oxide 2003;8:133-43.

18. Stachlewitz R, Seabra V, Bradford B et al. Glycine and uridine prevent D-galactosamine hepatotoxicity in the rat: Role of Kupffer cells. Hepatology 1999; 29:737-45.

19. Sun F, Hamagawa E, Tsutsui Ch et al. Evaluation of oxidative stress during apoptosis and necrosis caused by D-galactosamine in rat liver. Biochem Pharmacol 2003;65:101-7.

20. Yoshikawa T, Furukawa Y, Murakami M, Takemura S, Kondo M. Effects of vitamin $\mathrm{E}$ on D-galactosamine-induced or carbon tetrachloride-induced hepatotoxicity. Digestion 1982;25:222-9.

Submitted October 2005.

Accepted March 2006.

MUDr. Otto Kučera,

Charles Univesity in Prague, Faculty of Medicine in Hradec Králové, Deparment of Physiology, Simkova 870,

50038 Hradec Králové, Czech Republic. e-mail: kucerao@lfhk.cuni.cz 